Available online at www.sciencedirect.com

\title{
Interaction of Helix D of Elongation Factor Tu with Helices 4 and 5 of Protein L7/12 on the Ribosome
}

\author{
Ute Kothe, Hans-Joachim Wieden, Dagmar Mohr and \\ Marina V. Rodnina*
}

Institute of Physical

Biochemistry, University of

Witten/Herdecke, 58448 Witten

Germany

\begin{abstract}
Elongation factor $\mathrm{Tu}(\mathrm{EF}-\mathrm{Tu})$ promotes binding of aminoacyl-tRNA to the A site of the ribosome. Here, we report the effects of mutations in helix $\mathrm{D}$ of EF-Tu and in the C-terminal domain of L7/12 on the kinetics of A-site binding. Reaction rates were measured by stopped-flow and quench-flow techniques. The rates of A-site binding were decreased by mutations at positions 144, 145, 148, and 152 in helix D of EF-Tu as well as at positions $65,66,69,70,73$, and 84 in helices 4 and 5 of L7/12. The effect was due primarily to the lower association rate constant of ternary complex binding to the ribosome. These results suggest that helix D of EF-Tu is involved in an initial transient contact with helices 4 and 5 of L7/12 that promotes ternary complex binding to the ribosome. By analogy to the interaction of helix D of EF-Tu with the N-terminal domain of EF-Ts, the contact area is likely to consist of a hydrophobic patch flanked by two salt-bridges.
\end{abstract}

(C) 2004 Elsevier Ltd. All rights reserved.

Keywords: GTP-binding proteins; rapid kinetics; fluorescence; stopped flow; quench flow hydrolysis. ${ }^{7}$ Following the release of inorganic phosphate, the conformation of EF-Tu switches from the GTP to the GDP form, which has a greatly reduced affinity for aa-tRNA. Subsequently, aa-tRNA is released from EF-Tu.GDP, is accommodated in the $50 \mathrm{~S} \mathrm{~A}$ site, and takes part in peptide bond formation, while EF-Tu.GDP leaves the ribosome (Figure 1).

Little is known about the structural determinants in EF-Tu and the ribosome that are important for A-site binding. The only structures of the ternary complex bound to the ribosome available so far were determined by cryoelectron microscopy (cryo-EM). ${ }^{8-11}$ In these complexes, EF-Tu.GDP.aatRNA was blocked on the ribosome by the antibiotic kirromycin after GTP hydrolysis but before aa-tRNA was released from the factor. EF-Tu contacts the $50 \mathrm{~S}$ ribosomal subunit, while aa-tRNA spans the intersubunit cleft with the anticodon bound to the decoding center and the $3^{\prime}$ end of aa-tRNA attached to EF-Tu. The acceptor stem of aa-tRNA (nucleotide 69) contacts protein S12,11 whereas the T loop interacts with the L11-binding region on the $50 \mathrm{~S}$ subunit. ${ }^{10,11}$ The $\mathrm{G}$ domain of EF-Tu is located close to the ribosomal stalk and is involved in extensive interactions with the region underneath the stalk. According to biochemical 


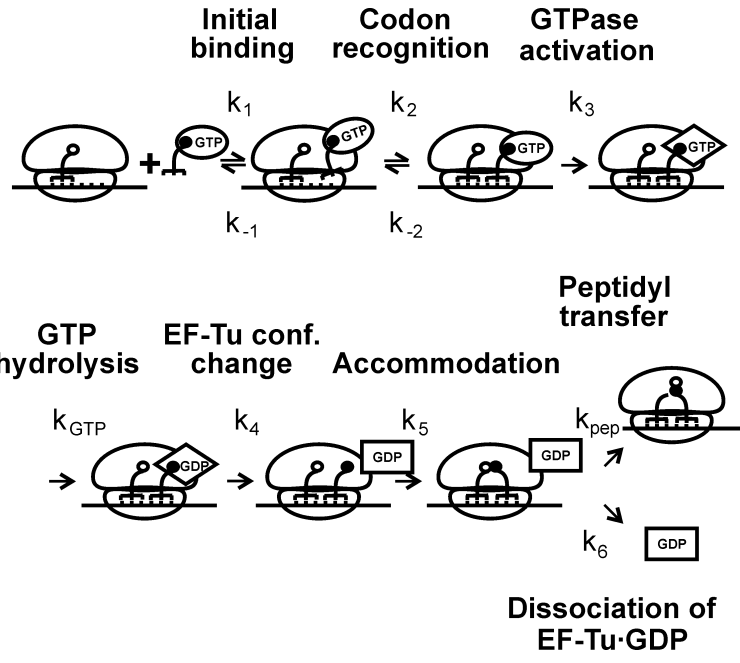

Figure 1. Mechanism of EF-Tu-dependent aa-tRNA binding to the A site. Kinetically resolved steps are indicated by rate constants $k_{1}-k_{6}$, steps that are rate-limited by the preceding step are denoted $k_{\mathrm{GTP}}$ and $k_{\text {pep }}$. EF-Tu is depicted differently in the GTP-bound and GDPbound conformations and in the GTPase-activated state.

data, immuno-electronmicroscopy, and modeling of the L7/12 proteins into the crystal structure of the $70 \mathrm{~S}$ ribosome, the stalk is comprised of the ribosomal proteins L7/12. ${ }^{12,13}$ The part of the ribosome underneath the stalk contains the sarcinricin loop of $23 \mathrm{~S}$ rRNA (SRL). ${ }^{9-11,13-15}$ A contact between the $G$ domain of EF-Tu, presumably around helix $\mathrm{D}$ of the factor, with $\mathrm{L} 7 / 12$ was suggested by cryo-EM at low resolution $(18 \AA) .{ }^{8}$ At higher resolution there was no clear density observed, although a contact between L7/12 and EF-Tu must exist at some point during A-site binding, as suggested by the biochemical evidence regarding the role of L7/12 in factor binding and functioning on the ribosome. ${ }^{16-18}$

In contrast to the EF-Tu-ribosome contacts, the interactions of EF-Tu with EF-Ts are well characterized by crystal structures. ${ }^{19,20}$ Helix D of EF-Tu was shown to bind to the N-terminal domain (NTD) of EF-Ts. ${ }^{20}$ Kinetic analysis revealed an important functional role of the interaction between helix D of EF-Tu and the NTD of EF-Ts in initiating nucleotide exchange in EF-Tu. ${ }^{21}$ Furthermore, sequence and structure comparisons of the NTD of EF-Ts and the C-terminal domain (CTD) of L7/12 suggested the presence of a similar surface patch, ${ }^{22}$ indicating that helix D may interact in the same way with EF-Ts and with L7/12 during EF-Tu-dependent A-site binding.

Here, we pursued the indications from the low-resolution cryo-EM ${ }^{8}$ and from structure and sequence alignments between EF-Ts and L7/12, ${ }^{22}$ and examined the role of helix $D$ in the $G$ domain of EF-Tu and the CTD of protein L7/12 for ternary complex binding to the ribosome. A number of amino acid residues in helix D of EF-Tu as well as in the CTD of L7/12 were exchanged for other amino acids, and the ability of the mutant proteins to promote aa-tRNA binding to the A site was studied. The data suggest that helix D of EF-Tu and helices $4 / 5$ of L7/12 are involved in the initial binding of the ternary complex to the ribosome. On the basis of mutagenesis and kinetic data, we propose a structural model for the interaction of helix D of EF-Tu with helices 4 and 5 of L7/12.

\section{Results}

\section{Effect of mutations in helix D of EF-Tu on ternary complex interactions with the ribosome}

To analyze the role of helix D of EF-Tu, a number of single amino acid exchanges were studied

(a)

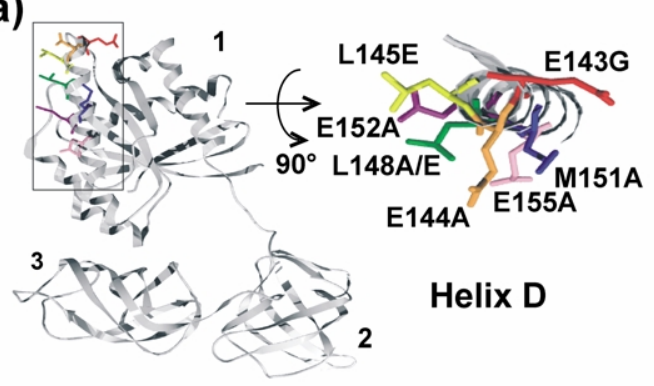

(b)

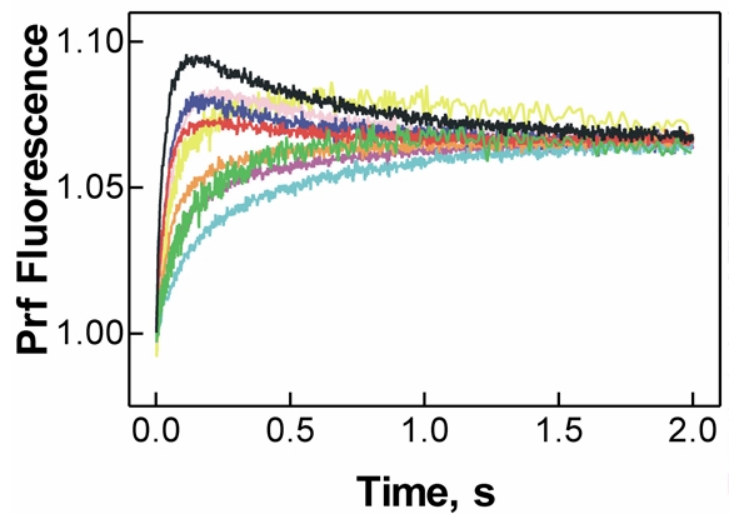

Figure 2. Effects of mutations in helix D of EF-Tu on aa-tRNA binding to the A site. (a) Positions of mutations. Left panel, crystal structure of EF-Tu in the EF-Tu.EF-Ts complex $^{20}$ from E. coli (PDB entry 1EFU); domains are numbered, helix D is boxed. Right panel, positions of mutations in helix D. (b) Time-courses of ternary complex binding to ribosomes. Ternary complex containing wild-type or mutant EF-Tu and Phe-tRNA ${ }^{\text {Phe }}(\operatorname{Prf16} / 17)$ (final concentration $0.1 \mu \mathrm{M}$ ) was mixed rapidly with poly(U)-programmed ribosomes containing AcPhetRNA $^{\text {Phe }}$ in the $\mathrm{P}$ site (final concentration $0.5 \mu \mathrm{M}$ ), and the fluorescence of proflavin was monitored. Color code for the mutated residues is the same in (a) and (b): black, wild-type EF-Tu (the apparent rate constants calculated by two-exponential fitting were $33 \mathrm{~s}^{-1}$ and $\left.2 \mathrm{~s}^{-1}\right)$; EF-Tu mutants: red, E143G $\left(25 \mathrm{~s}^{-1}\right.$ and $\left.3 \mathrm{~s}^{-1}\right)$; orange, E144A $\left(18 \mathrm{~s}^{-1}\right.$ and $\left.3 \mathrm{~s}^{-1}\right)$; yellow, L145E $\left(17 \mathrm{~s}^{-1}\right.$ and $\left.0.1 \mathrm{~s}^{-1}\right)$; turquoise, L148A $\left(10 \mathrm{~s}^{-1}\right.$ and $\left.2 \mathrm{~s}^{-1}\right)$; green, L148E $\left(20 \mathrm{~s}^{-1}\right.$ and $\left.5 \mathrm{~s}^{-1}\right)$; blue, M151A $\left(23 \mathrm{~s}^{-1}\right.$ and $\left.3 \mathrm{~s}^{-1}\right)$; purple, E152A $\left(14 \mathrm{~s}^{-1}\right.$ and $\left.2 \mathrm{~s}^{-1}\right)$; and pink, E155A $\left(17 \mathrm{~s}^{-1}\right.$ and $\left.2 \mathrm{~s}^{-1}\right)$. 
(Figure 2(a)). Mutant proteins containing a C-terminal oligohistidine tag were expressed and purified as described. ${ }^{21}$ The positions of mutations included the exposed, strictly conserved surface residues E144, L145, L148, and E152, and several less conserved, solvent-exposed positions (E143, M151, E155). Residues oriented towards the interior of EF-Tu were not replaced, because mutations of the respective side-chains are likely to disturb the overall structure of the region. Mutations in helix $\mathrm{D}$ did not affect the ability of EF-Tu to bind GTP and aa-tRNA, as verified by gel-filtration studies ${ }^{23}$ and by assaying the protection of the aminoacyl ester bond of aa-tRNA against hydrolysis (data not shown). ${ }^{24}$ All EF-Tu mutants were able to promote full aa-tRNA binding to the ribosome within one minute of incubation; hence, only kinetic effects could be expected. In the following, the effect of helix D mutations on the partial reactions of A-site binding (Figure 1) was analyzed using rapid kinetic methods.

The interaction of the ternary complex EFTu.GTP.Phe-tRNA ${ }^{\text {Phe }}(\operatorname{Prf16} / 17)$ with poly(U)-programmed ribosomes was monitored by changes in proflavin fluorescence. As shown previously, ${ }^{23}$ binding of the wild-type ternary complex to the ribosome leads to a biphasic fluorescence change (Figure 2(b)) reflecting ternary complex binding to the ribosome and codon recognition (fluorescence increase) and a rearrangement towards the final A site-bound state of the tRNA (fluorescence decrease) taking place after GTP hydrolysis (accommodation). Ternary complexes with mutations in positions 143,145, 151, and 155 of EF-Tu exhibited fluorescence changes similar to that of the wild-type ternary complex, although amplitudes were decreased by mutations E143G and E155A. With mutants E144A, L148A, L148E, and E152A, the fluorescence change was qualitatively different (Figure 2(b)). Only an increase in proflavin fluorescence was observed, while the highfluorescence intermediate formed with wild-type EF-Tu and the other mutants could not be detected. In the following, rate constants of the A-site binding were determined for one of the mutants showing altered behavior, EF-Tu(E152A), in order to identify the step that was affected by the mutation.

\section{Rate constants of A-site binding of EF-Tu(E152A).GTP.Phe-tRNA ${ }^{\text {Phe }}$}

Rate constants of the elemental steps of A-site binding (Figure 1) were determined as outlined below. ${ }^{6,25}$ The kinetic analysis was performed by fluorescence stopped flow using fluorescent derivatives of $t R N A$, Phe-tRNA ${ }^{\text {Phe }}(\operatorname{Prf16} / 17),{ }^{3,23}$ or GTP, 2'-(or 3')-O-(N-methylanthraniloyl) (mant)GTP. ${ }^{7}$ Rates of peptide bond formation were measured by quench flow. Rate constants obtained with EF-Tu(E152A) were compared to those measured under the same conditions with wildtype EF-Tu containing an oligohistidine tag. ${ }^{25}$
Kinetics of codon-independent initial binding were measured using non-programmed ribosomes and ternary complexes containing fluorescent PhetRNA $^{\text {Phe }}($ Prf16/17). Binding of the ternary complex to non-programmed ribosomes leads to a rapid exponential fluorescence increase. Upon increasing the ribosome concentration, the rate of association increased linearly (Figure 3(a)). From the concentration-dependence of the apparent rate constant,

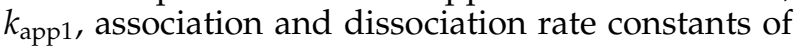
initial binding, $k_{1}$ and $k_{-1}$, were determined. The values of the rate constants $k_{1}$ and $k_{-1}$ were $100( \pm 10) \mu \mathrm{M}^{-1} \mathrm{~s}^{-1}$ and $13( \pm 7) \mathrm{s}^{-1}$, respectively, with the ternary complex containing wild-type EF-Tu with an oligohistidine tag. ${ }^{25}$ With EF$\mathrm{Tu}(\mathrm{E} 152 \mathrm{~A}), k_{1}$ was reduced to $50( \pm 10) \mu \mathrm{M}^{-1} \mathrm{~s}^{-1}$, while the $k_{-1}$ value, $13( \pm 10) \mathrm{s}^{-1}$, was not changed (Table 1). Thus, the association rate constant of the ternary complex to the ribosome was affected by the mutation in helix D.

To determine the elemental rate constants of the following steps of A-site binding, time-courses such as those shown in Figure 2(b) were measured at increasing concentration (up to $4 \mu \mathrm{M}$ ) of poly(U)-programmed $\mathrm{P}$ site-blocked ribosomes. Time-courses were analyzed by two-exponential fitting. Notably, at concentrations of poly(U)-programmed ribosomes higher than $0.5 \mu \mathrm{M}$ (Figure 2(b)), time-courses obtained with EF-Tu(E152A) were similar in shape to those with wild-type EF-Tu, because the high-fluorescence intermediate consumed in a following reaction became visible (data not shown). This can be explained by kinetic differences between wild-type and mutant EF-Tu. At low concentrations of ribosome, initial binding was slow and rate-limiting for codon recognition. Therefore, the high-fluorescence intermediate codon-recognition complex was consumed rapidly by the fluorescence decrease accompanying accommodation, reflected in the shape of the time-course (Figure 2(b)). At higher concentrations of ribosome, initial binding became faster, resulting in significant accumulation of the highfluorescence codon-recognition complex.

As assigned previously, the first exponential term reflects the apparent rate constant of codon recognition, whereas the second reflects a reaction that takes place after GTP hydrolysis, i.e. aa-tRNA accommodation in the A site. ${ }^{6,25}$ The apparent rate constant of the faster step, $k_{\text {app2 }}$, increased hyperbolically with ribosome concentration and saturated at $65( \pm 5) \mathrm{s}^{-1}$ (Figure $3(\mathrm{~b})$ ), consistent with a first-order rearrangement (codon recognition) following the second-order association step (initial binding). The value of $k_{-2}$ was not measured with EF-Tu(E152A); mutations in EF-Tu were not expected to alter this value appreciably, because the dissociation of the codon recognition complex is governed mainly by the complementarity of the codon-anticodon complex. At the present experimental condition, a value of $k_{-2}$ around $0.2 \mathrm{~s}^{-1}$ is expected. ${ }^{26}$

The rearrangement leading to GTPase activation 
(a)

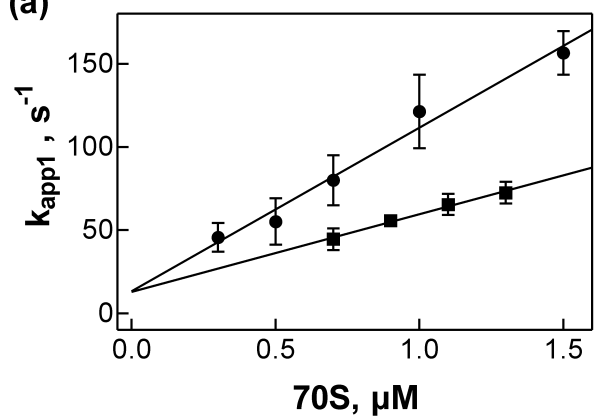

(c)

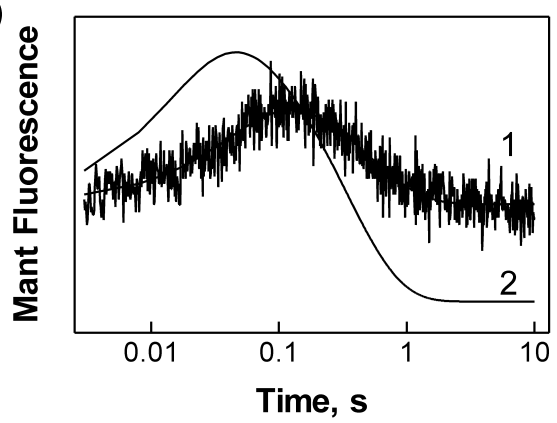

(b)

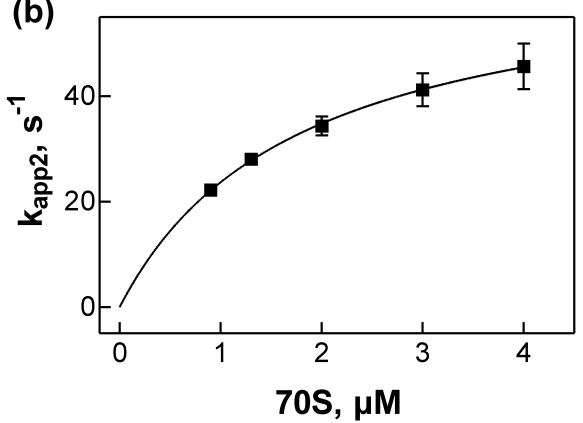

(d)

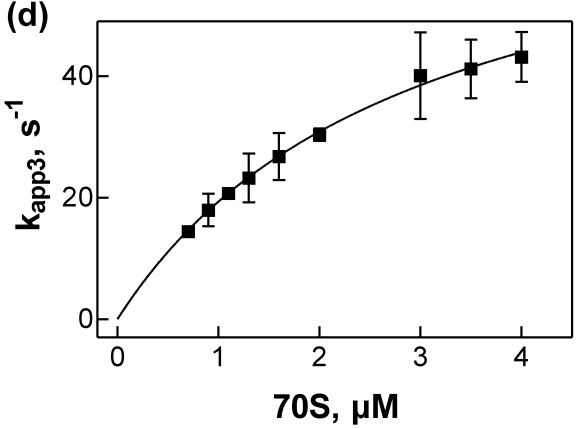

(e)

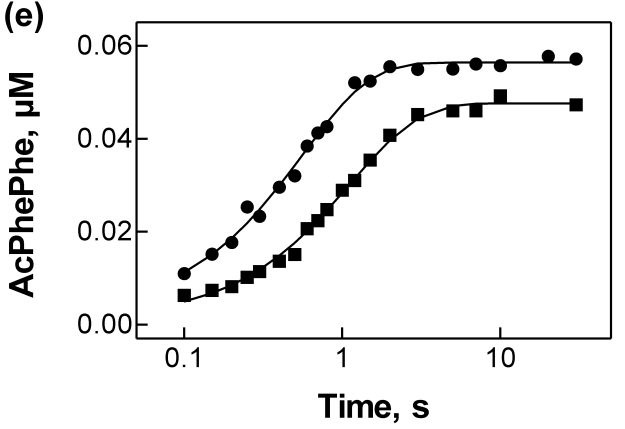

Figure 3. Kinetic analysis of A-site binding of EF-Tu(E152A).GTP.Phe-tRNA ${ }^{\text {Phe }}$. (a) Initial binding measured with wild-type $\mathrm{EF}^{-\mathrm{Tu}^{25}}$ (circles) or EF-Tu(E152A) (squares). Apparent rate constants were determined by exponential fitting of the time-courses of the fluorescence changes measured after mixing the ternary complexes EF-Tu(E152A).GTP.PhetRNA $^{\text {Phe }}($ Prf16/17) $(0.2 \mu \mathrm{M})$ with increasing amounts of non-programmed ribosomes in the stopped-flow apparatus. (b) Concentration-dependence of the apparent rate constant of codon recognition. Fluorescence changes of proflavin in tRNA $^{\text {Phe }}$ (Prf16/17) were measured after mixing ternary complex containing EF-Tu(E152A) $(0.2 \mu M)$ with increasing concentrations of poly $(\mathrm{U})$-programmed $\mathrm{P}$ site-blocked ribosomes. (c) Conformational changes of EF-Tu measured by mant-GTP fluorescence changes after mixing of ternary complex containing EF-Tu(E152A) $(0.2 \mu \mathrm{M})$ with poly(U)-programmed ribosomes $(0.9 \mu \mathrm{M})$ (transient and fit 1$)$. The fit of the analogous curve obtained with wild-type EF-Tu is shown for comparison (fit 2). Two-exponential fitting yielded $60( \pm 10) \mathrm{s}^{-1}$ and $3.0( \pm 0.3) \mathrm{s}^{-1}$ for wild-type EF-Tu, and $20( \pm 4) \mathrm{s}^{-1}$ and $2.0( \pm 0.3) \mathrm{s}^{-1}$ for EF-Tu(E152A). (d) Concentration-dependence of the apparent rate constant of GTPase activation. The $k_{\text {app }}$ values were determined from time-courses measured by mant fluorescence upon mixing of $\mathrm{EF}-\mathrm{Tu}(\mathrm{E} 152 \mathrm{~A}) \cdot \mathrm{mant}^{-G T P} \cdot \mathrm{Phe}-\mathrm{tRNA}{ }^{\text {Phe }}(0.2 \mu \mathrm{M})$ with increasing concentrations of poly(U)-programmed ribosomes. (e) Peptide bond formation. Time-courses of AcPhePhe formation were measured by quench-flow upon mixing ternary complexes containing Phe-tRNA ${ }^{\text {Phe }}$ and either wild-type EF-Tu (circles) or EF-Tu(E152A) (squares) (0.2 $\left.\mu \mathrm{M}\right)$ with

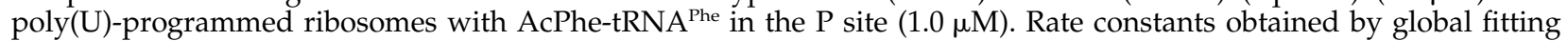
(as described in Materials and Methods) were 1.8( \pm 0.2$) \mathrm{s}^{-1}$ and 1.0 $( \pm 0.2) \mathrm{s}^{-1}$ for wild-type EF-Tu and EF-Tu(E152A), respectively.

in $\mathrm{EF}-\mathrm{Tu}(\mathrm{E} 152 \mathrm{~A})$ was monitored by fluorescence changes of mant-GTP, measured at increasing concentrations of poly(U)-programmed ribosomes. Similar to the situation with wild-type EF-Tu, ${ }^{7,25}$ the interaction of EF-Tu(E152A)-mant-GTP.PhetRNA $^{\text {Phe }}$ with the ribosome resulted in a biphasic fluorescence change (Figure 3(c)). The increase in mant-fluorescence, characterized by the apparent rate constant $k_{\mathrm{app} 3}$, reflects the GTPase activation rearrangement within the $G$ domain of EF-Tu which precedes, and limits the rate of, GTP hydrolysis. Subsequent steps, comprising GTP hydrolysis, the change of EF-Tu from the activated to the GDP conformation, and the release of 
Table 1. Effect of the E152A mutation in helix D of EF-Tu on the elemental rate constants of ternary complex binding to the A site

\begin{tabular}{|c|c|c|c|}
\hline \multirow[t]{2}{*}{ Step ${ }^{a}$} & & \multicolumn{2}{|c|}{ Rate constant $\left(\mathrm{s}^{-1}\right)$} \\
\hline & & Wild-type ${ }^{\mathrm{b}}$ & E152A \\
\hline \multirow[t]{2}{*}{ Initial binding } & $k_{1}$ & $100 \pm 10^{c}$ & $50 \pm 10^{c}$ \\
\hline & $k_{-1}$ & $13 \pm 7$ & $13 \pm 10$ \\
\hline Codon recognition $^{\mathrm{d}}$ & $k_{2}$ & $140 \pm 50$ & $60 \pm 10$ \\
\hline GTPase activation and GTP hydrolysis ${ }^{\mathbf{e}}$ & $k_{3}$ & $>500$ & $>400$ \\
\hline Conformational change of EF-Tu & $k_{4}$ & $25 \pm 10$ & $8 \pm 7$ \\
\hline Aa-tRNA accommodation and peptide bond formation ${ }^{e}$ & $k_{5}$ & $1.8 \pm 0.2^{\mathrm{f}}$ & $1.0 \pm 0.2$ \\
\hline Dissociation of EF-Tu & $k_{6}$ & $3 \pm 1$ & $2 \pm 1$ \\
\hline $\begin{array}{l}\text { a Steps are defined according to the model in Figure } 1 \\
\text { b Determined previously using EF-Tu with an oligohis } \\
\text { c Units are } \mu \mathrm{M}^{-1} \mathrm{~s}^{-1} \text {. } \\
\text { d The value of the dissociation rate constant of the coc } \\
\text { e Steps grouped for kinetic analysis, because the form } \\
\text { f Determined from Figure } 3(\mathrm{e}) \text {. }\end{array}$ & $\begin{array}{l}\text { com } \\
\text { ate-lin }\end{array}$ & $\begin{array}{l}\text { ot determin } \\
\text { tter. }\end{array}$ & \\
\hline
\end{tabular}

aa-tRNA from EF-Tu, do not influence the fluorescence. The steps of GTPase activation and GTP hydrolysis appear as one kinetic step, because GTP hydrolysis is rate-limited by the preceding GTPase activation step. ${ }^{6,26}$ The decrease in mantfluorescence reports the dissociation of EFTu-mant-GDP from the ribosome. ${ }^{6}$

The formation of the high-fluorescence intermediate was slower and the amplitude of the fluorescence change was decreased with EF$\mathrm{Tu}(\mathrm{E} 152 \mathrm{~A})$, compared to wild-type EF-Tu (Figure 3(c)). At limiting concentrations of ribosome, slower GTPase activation resulted from the lower rate of initial binding to the ribosome of ternary complex containing EF-Tu(E152A). From the hyperbolic fit of the concentration-dependence, the $k_{\text {app3 }}$ value at saturation was $75( \pm 10) \mathrm{s}^{-1}$ (Figure 3(d)), i.e. identical with the rate constant of codon recognition $\left(k_{\mathrm{app} 2}=65( \pm 5) \mathrm{s}^{-1}\right.$, cf. above) within the standard deviation. Thus, GTPase activation in EF-Tu(E152A) is rapid, and its rate cannot be determined with precision. The rate of EF-Tu.GDP dissociation from the ribosome, $k_{6}$, was determined from the decrease of the mantGDP fluorescence. ${ }^{6}$ The rate constant of dissociation was $2( \pm 1) \mathrm{s}^{-1}$ and independent of the concentration of ribosome (Table 1). Dissociation of EF-Tu.GDP took place at a comparable rate with wild-type EF-Tu and EF-Tu(E152A), indicating that dissociation of EF-Tu.GDP was not affected by the mutation.

The rate of aa-tRNA accommodation in the $A$ site, $k_{5}$, was determined from the time-courses of dipeptide formation (Figure 3(e)). These two steps are not distinguished kinetically, because the chemistry step is limited by the rate of the preceding accommodation step. ${ }^{6,26}$ The time-course of dipeptide formation was measured by quench flow upon binding of ternary complex containing $\left[{ }^{14} \mathrm{C}\right]$ Phe-tRNA ${ }^{\text {Phe }}$ to the A site of poly(U)-programmed ribosomes carrying AcPhe-tRNA ${ }^{\text {Phe }}$ in the $\mathrm{P}$ site. Peptide bond formation took place after a pronounced lag phase that comprised the steps preceding peptide bond formation (Figure 1). The rates of peptide bond formation were similar for wild-type and mutant ternary complexes, $1.8 \mathrm{~s}^{-1}$ and $1 \mathrm{~s}^{-1}$, respectively, suggesting that dipeptide formation is not affected significantly by the E152A mutation. The rate constant of the conformational rearrangement of EF-Tu from the GTP to the GDP-form, $k_{4}$, was calculated from the delay phase of the time-courses of peptide bond formation, taking into account the rates of all steps contributing to the delay, i.e. initial binding, codon recognition, GTPase activation, and the transition from the GTP to the GDP-form. ${ }^{6,25,26}$ The difference in the $k_{4}$ values between wild-type and mutant EF-Tu was not significant, given relatively large standard deviations of the values (Table 1).

As summarized in Table 1 , the association rate constant of initial binding was reduced twofold by the mutation E152A, while the dissociation rate constant $k_{-1}$ was not affected. The rate constant of codon recognition was decreased by a factor of 2 . No difference between wild-type and mutant EF-Tu was found for the rate constant of GTPase activation and all subsequent steps of A-site binding, i.e. GTP hydrolysis, conformational change of EF-Tu, aa-tRNA accommodation in the A site, peptide bond formation, and EF-Tu.GDP dissociation from the ribosome. Thus, the mutation E152A in helix D of EF-Tu predominantly affects the initial steps of A-site binding.

\section{Localization of the ribosome-contact area in helix D of EF-Tu}

To localize the interaction surface in helix D of EF-Tu and quantify the effects of mutations on A-site binding, we analyzed the effect of several single amino acid substitutions (Figure 2(a)) on the rate constants of initial binding. For all mutants of EF-Tu, association and dissociation rate constants, $k_{1}$ and $k_{-1}$, were determined by stoppedflow measurements performed at increasing 

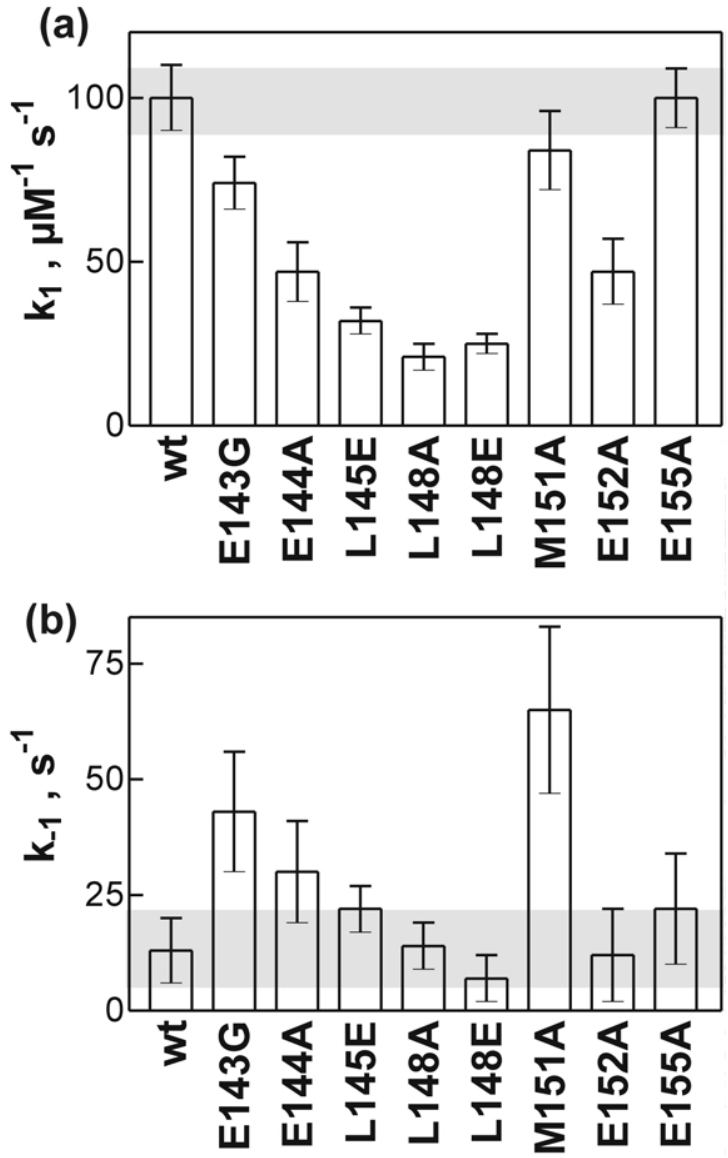

Figure 4. Effect of mutations in helix D of EF-Tu on ternary complex binding to the ribosome. (a) Association rate constant, $k_{1}$. (b) Dissociation rate constant, $k_{-1}$. The range of rate constants obtained for wild-type EF-Tu is highlighted in grey. Time-courses were measured with ternary complexes containing Phe-tRNA ${ }^{\text {Phe }}(\operatorname{Prf16} / 17)$ $(0.2 \mu \mathrm{M})$ and various amounts of non-programmed ribosomes $(0.75-2.0 \mu \mathrm{M}) . k_{\mathrm{app}}$ values were determined by exponential fitting, and $k_{1}$ and $k_{-1}$ were calculated from the slopes and intercepts, respectively, of the concentration-dependence of $k_{\text {app }}$ (see Figure 3(a)).

concentration of ribosome. The effect of helix D mutations on $k_{1}$ and $k_{-1}$ is shown in Figure 4.

The mutations E144A, L145E, L148A/E, and E152A decreased the association rate constant, $k_{1}$, about two- to fourfold, compared to wild-type EF-Tu, whereas the dissociation rate constant, $k_{-1}$, was not affected. Mutation E155A had no effect. Mutations E143G and M151A had little influence on the association rate constant, whereas the dissociation rate constant was increased, indicating a lower level of stability of these complexes. The data suggest that positions 144, 145, 148 and 152 in helix D of EF-Tu are involved in transient initial binding of the ternary complex to the ribosome.

\section{Localization of the EF-Tu contact area in the CTD of L7/12}

To identify amino acid residues in the ribosomal protein L7/12 taking part in the interaction with

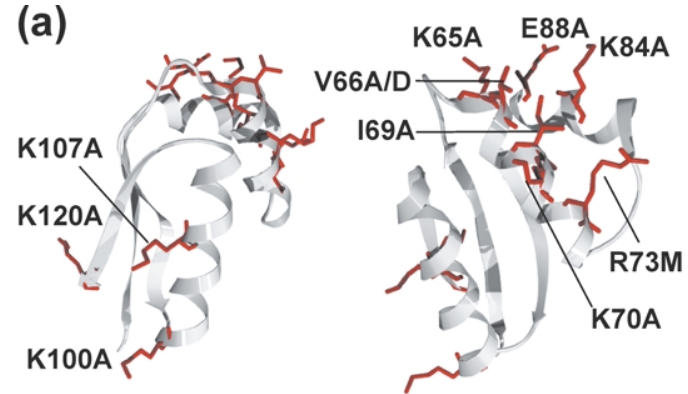

(b)

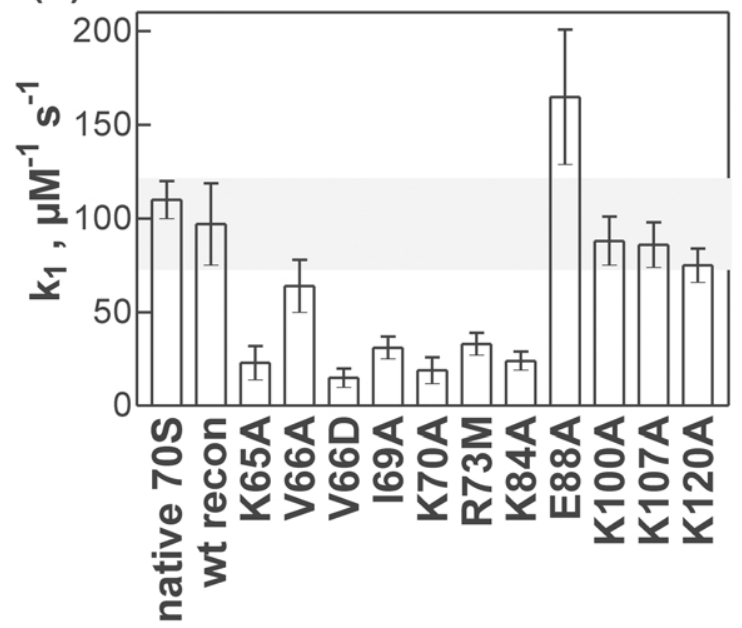

(c)

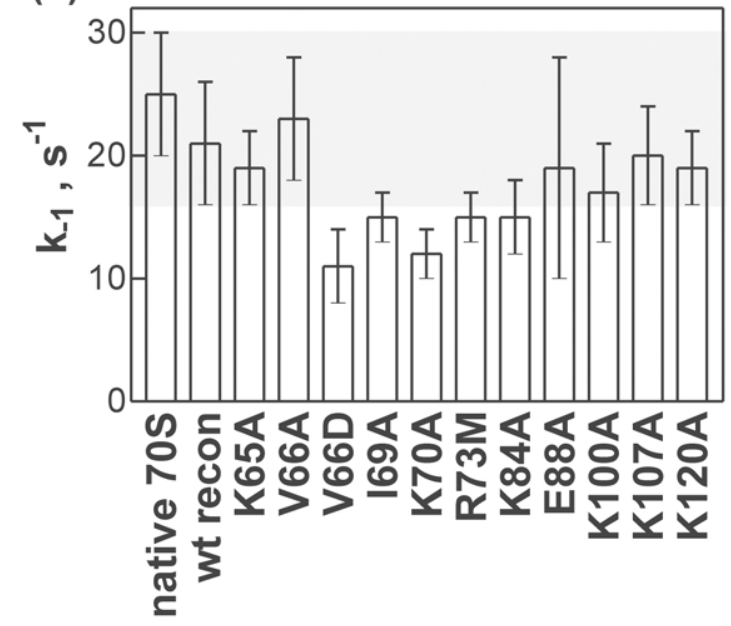

Figure 5. Effect of mutations in L7/12 on ternary complex binding to the ribosome. (a) Position of mutations in L7/12. Left and right panels, crystal structure of the CTD of L7/12 from E. coli $\left(1 \mathrm{CTF}^{30}\right)$; the views differ by a $90^{\circ}$ rotation along the vertical axis. (b) Association rate constant, $k_{1}$. (c) Dissociation rate constant, $k_{-1}$. The range of rate constants obtained for native ribosomes and ribosomes reconstituted with wild-type L7/12 is highlighted in grey. Time-courses were measured with ternary complexes containing Phe-tRNA ${ }^{\text {Phe }}(\operatorname{Prf16} / 17)(0.05 \mu \mathrm{M})$ and non-programmed ribosomes $(0.15-1.0 \mu \mathrm{M})$ reconstituted with different L7/12 mutants. The $k_{\text {app }}$ values were determined by exponential fitting; $k_{1}$ and $k_{-1}$ were calculated from the slopes and intercepts, respectively, of the linear concentration-dependence of $k_{\text {app }}$. 
the ternary complex, we analyzed the effect on initial binding of single amino acid substitutions in the three $\alpha$ helices in the CTD of L7/12 (Figure $5(\mathrm{a})$ ). Helix 4 of the CTD contains a number of strictly conserved residues; the solvent-exposed residues were mutated (K65, V66, I69, K70, R73). In helix 5 , the only strictly conserved surface residue is K84, which was mutated. Helix 5 also contains a number of charged surface residues that are less conserved; of those positions, E88 was chosen to introduce a mutation. Other parts of the CTD are less well conserved. Three charged surface residues that are $>80 \%$ identical and located on the side opposite of helices 4 and 5 were mutated (K100, K107, and K120). Mutagenesis was performed and mutant L7/12 proteins were expressed and purified as described. ${ }^{27}$ To incorporate mutant L7/12 into ribosomes, ribosomes were first depleted of $\mathrm{L} 7 / 12$ and then reconstituted with mutant L7/12 added in excess. ${ }^{18}$ Initial binding of ternary complex to ribosomes containing mutant L7/12 was monitored by fluorescence stopped-flow. Association and dissociation rate constants, $k_{1}$ and $k_{-1}$, are summarized in Figure 5.

Ribosomes reconstituted with wild-type L7/12 showed the same rate constants of initial binding, $k_{1}$ and $k_{-1}$, as untreated, native ribosomes. Thus, L7/12 depletion and reconstitution does not influence the kinetics of initial binding. Mutations in positions 65-84 of L7/12 decreased the association rate constant of initial binding, $k_{1}$, about fourfold, compared to native ribosomes (Figure 5(b)). The V66A substitution had a smaller effect, i. e. a twofold decrease in $k_{1}$. Interestingly, the mutation E88A increased $k_{1}$. The dissociation rate constant, $k_{-1}$, was hardly affected by helix $4 / 5$ mutations (Figure 5(c)). Mutations K100A, K107A and K120A did not influence $k_{1}$ or $k_{-1}$. These data suggest that helices 4 and 5 in the CTD of L7/12 are involved in the interaction with the ternary complex during initial binding.

\section{Discussion}

\section{Role of helix D of EF-Tu in binding of the ternary complex to the ribosome}

The present data show that replacement of amino acid residues in helix D of EF-Tu at positions E144, L145, L148 and E152 results in a decrease of the association rate constant, $k_{1}$, of ternary complex binding to the ribosome, suggesting an important role of helix $\mathrm{D}$ in the interaction. Mutations at positions 143, 151 and 155 did not influence $k_{1}$. The side-chains of E143 and M151 point to a different direction than those of E144, L145, L148 and E152, indicating that the interaction surface on helix D is limited to the side where the latter residues are located. E155 is found in the C-terminal region of helix D, suggesting that this region is not involved in the contact. The increase in the dissociation rate constant, $k_{-1}$, observed for
EF-Tu mutants E143G and M151A may result from a somewhat different structure or orientation of helix $\mathrm{D}$ in these mutants, compared to wildtype EF-Tu, which lowers the stability of the initial binding complex.

For EF-Tu(E152A), which showed a strong effect on the kinetics of A-site binding, the rate constants of all further steps of A-site binding were determined. The rate constant of codon recognition, $k_{2}$, was decreased twofold by the E152A mutation. Slower binding may be explained either by indirect effects, such as incorrect positioning of aa-tRNA in the initial binding complex as a result of the mutation in EF-Tu, or by a specific role of interactions of helix $\mathrm{D}$ with the ribosome in the adjustment of the ternary complex during codon recognition. GTPase activation of EF-Tu and the following steps of A-site binding were not decreased appreciably by the E152A mutation. In summary, helix D of EF-Tu seems to be involved in the initial contact between the ternary complex and the ribosome, rather than in subsequent steps of A-site binding, including GTPase activation.

\section{Helix D of EF-Tu may interact in a similar way with EF-Ts and L7/12}

The kinetic effects of L7/12 mutations can be used to map the surface on $\mathrm{L} 7 / 12$ for the interaction with EF-Tu. The present data show that all mutations in L7/12 that affect initial binding are located in helices 4 and 5 . This is consistent with earlier reports indicating that mutations in this region of $\mathrm{L} 7 / 12$ decrease both rate and accuracy of translation, ${ }^{28,29}$ most likely due to an impaired function of EF-Tu. The decrease in the rate constant of initial binding due to mutations in L7/12, up to fivefold, was comparable to the effect caused by complete removal of $\mathrm{L} 7 / 12,{ }^{18}$ indicating a strong impairment of binding by the mutations. Furthermore, the results of the present mutagenesis studies support the contention of an interaction between helix D of EF-Tu and helices 4 and 5 of L7/12 that was deduced from sequence-based and structure-based alignments of L7/12 and EF-Ts, ${ }^{22}$ as described in detail below. On the basis of these results, we propose that during initial binding of ternary complex to the ribosome, helix D of EF-Tu forms a contact to helices 4 and 5 of protein L7/12 in a way similar to that in the EF-Tu.EF-Ts complex (Figure 6).

The interaction surface between EF-Tu and L7/12 was predicted to consist of a hydrophobic patch formed by interactions between the sidechains of L145, L148, and V149 of EF-Tu and K70, L80, and V66 of L7/12, hydrophobic interaction between V140 and L145 of EF-Tu and K81 in L7/12, as well as two salt-bridges, E152 in EF-Tu to R73 in L7/12 and D141 in EF-Tu (outside helix D) to K84 in L7/12 (Figure 6(a)). ${ }^{22}$ In accordance with the model, mutations of residues expected to form the hydrophobic patch, L145A, L148A, L148E in EF-Tu and K70A, V66A, V66D in L7/12 had an 
(a)

EF-Tu

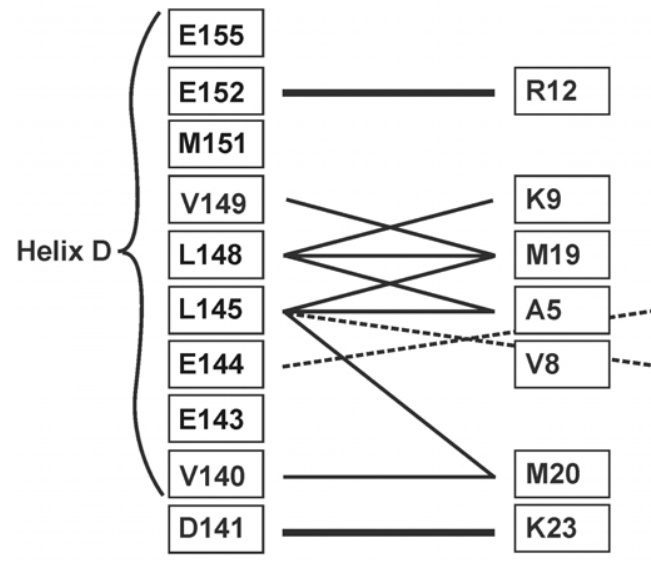

(b)

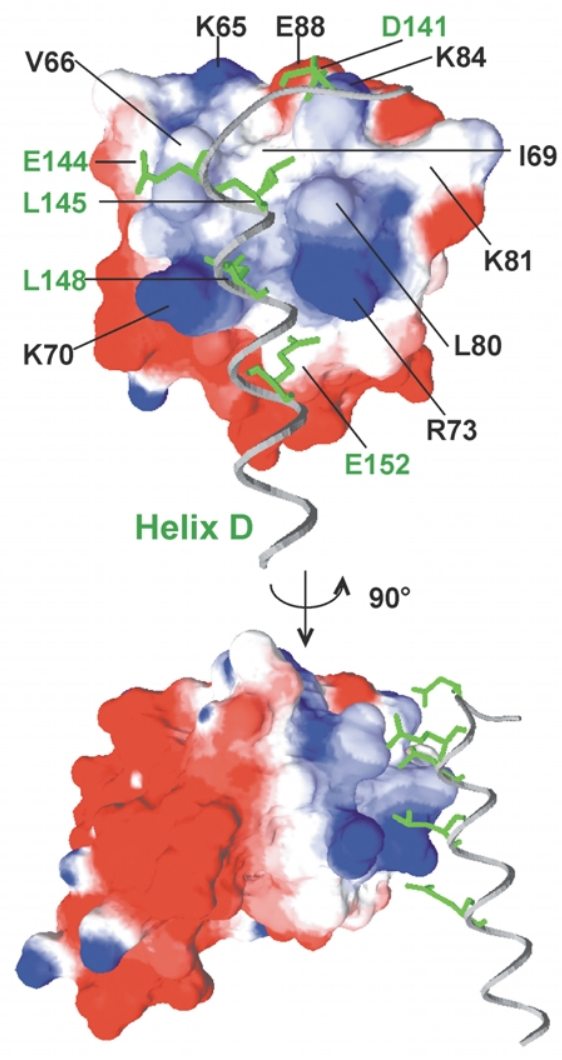

Figure 6. Interactions between helix D of EF-Tu and the CTD of L7/12. (a) Scheme of putative contacts. The binding pattern between helix D of EF-Tu and EF-Ts was taken from the crystal structure of the EF-Tu.EF-Ts complex ${ }^{20}$ the sequence alignment of EF-Ts and L7/12 is from Wieden et al. ${ }^{22}$ Contacts that are similar in the EF-Tu.L7/12 and EF-Tu.EF-Ts complexes are shown as thin (hydrophobic interactions) and thick (salt-bridges) lines. Broken lines indicate interactions between EF-Tu and L7/12 suggested by the present data, which are not found in the EF-Tu.EF-Ts complex. (b) Structural model. Helix D of EF-Tu (depicted as ribbon) is taken from the EF-Tu.EF-Ts crystal structure $\left(1 \mathrm{EFU}^{20}\right)$. The CTD of L7/12 $\left(1 \mathrm{CTF}^{30}\right)$ is shown in surface representation colored according to charge (red, negative; blue, positive). The position of L7/12 in the complex with EF-Tu was effect on ternary complex binding to the ribosome. Mutation of L148 in EF-Tu resulted in a fourfold reduction of the association rate constant. The effect was observed with exchanges of L148 to both Ala and Glu, indicating that both hydrophobicity and size of Leu are important at this position. In the model, Leu148 forms the core of a hydrophobic patch in the middle of the interaction surface (Figure 6). The L148E replacement introduced a negative charge into that patch, disturbing the hydrophobic interaction, while Ala appears to be too small for the tight packing of the interaction surfaces. Similarly, the L145E mutation resulted in a threefold decrease in the association rate constant due to interference of the charge with the hydrophobic patch. Replacement of Val66 by Asp in L7/12 resulted in a fivefold decrease of the association rate constant, whereas a more conservative replacement to Ala reduces $k_{1}$ by a factor of only 2. The I69A mutation in L7/12 also decreased the association constant about threefold. I69 can be aligned to V8 in EF-Ts (Figure 6(a)), a residue that was not implicated in the EF-Tu.EF-Ts contacts. ${ }^{20}$ However, the position and orientation of I69 in the groove between helices 4 and 5 of the CTD of L7/12 30,31 (Figure 6(b)) would be consistent with the involvement of this residue in the formation of the hydrophobic interaction between EF-Tu and L7/12.

Residues L148 and E152 in helix D of EF-Tu are important for both the binding of the ternary complex to the ribosome (present results) and interactions with the NTD of EF-Ts. ${ }^{21}$ In addition to their role in binding EF-Tu to EF-Ts, L148 and E152 of EF-Tu were found to be important for the acceleration of GDP dissociation from the EF-Tu.EF-Ts complex, probably by introducing a distortion at the base binding site. ${ }^{21}$ However, the E152A mutation had no effect on GTPase activation and subsequent steps of A-site binding (Table 1), suggesting that, although helix $\mathrm{D}$ is important for the initial contact with both EF-Ts and L7/12, the functional consequences of contacts with helix $\mathrm{D}$ are different in the two cases.

E152 was modeled to form a salt-bridge to R73 of L7/12 (Figure 6). In fact, mutations in both E152 in EF-Tu and R73 in L7/12 decreased the association rate constant two- and fourfold, respectively. The deficiency of the R73 mutant in initial binding of ternary complex to the ribosome explains the decrease of translational efficiency upon chemical modification of R73. ${ }^{32,33}$ Likewise, mutation of K84 of $\mathrm{L} 7 / 12$, a residue expected to form a salt-bridge to EF-Tu, resulted in a fivefold decrease of the binding rate constant. K81 of L7/12 which, by analogy to M20 of EF-Ts is expected to form a hydrophobic interaction with V140 of EF-Tu, was not mutated, because this residue is not strictly conserved among L7/12 proteins.

obtained by alignment of helices 4 and 5 with helices 1 and 2 of EF-Ts. ${ }^{22}$ Residues involved in the interaction are indicated (L7/12, black; EF-Tu, green). 
There are three other residues (E144 of EF-Tu; K65 and E88 of L7/12) that also affect the binding of the ternary complex to the ribosome, suggesting additional interactions between EF-Tu and L7/12 that are not present in the EF-Tu.EF-Ts complex. ${ }^{20}$ The function of these residues can be deduced from the structural alignment of helices $4 / 5$ of L7/12 and helices $1 / 2$ of EF-Ts. The hydrophobic part of the side-chain of E144 is packed against V66 and contributes to the hydrophobic patch. The contact may be stabilized further by electrostatic attraction between E144 and the respective part of helix 4 in L7/12, which is positively charged (Figure 6(b)). In the EF-Tu.EF-Ts complex, this contact is not present, possibly because in EF-Ts alanine is found at position 5, which is equivalent to V66 of L7/12.

The side-chains of $\mathrm{K} 65$ and E88 in L7/12 are located too far away to be involved in direct contacts with helix D of EF-Tu. Nevertheless, these residues could influence the interaction with helix $\mathrm{D}$ by charge effects. Compared to the overall acidic nature of L7/12, helices 4 and 5 are positively charged, due to the presence of four lysine residues and one arginine residue (K65, K70, R73, K81 and K84). The presumed complementary surface in helix D is negatively charged, especially in the N-terminal region (D141, D142, E143, E144, E147, E150, E152). Therefore, K65 may contribute to the attraction or preorientation of helix D to helices 4 and 5 of L7/12. Hence, reduction of the positive charge by the $\mathrm{K} 65 \mathrm{~A}$ mutation could be the reason for the decreased rate constant of ternary complex binding to the ribosome. Likewise, removal of the negative charge of E88 in the same region of L7/12 could increase the attraction of helix D and cause the observed increase in the association rate constant. Alternatively, K65 might form a salt-bridge with D142 of EF-Tu, which may be positioned within a distance of $5 \AA$ by a slight movement of helix D from its orientation in Figure 6(b).

In summary, the effects of mutations in helix D of EF-Tu and helices 4 and 5 of L7/12 are consistent with the binding model based on the structure similarity between L7/12 and EF-Ts. ${ }^{22}$ In both EF-Ts and L7/12, the structural motif seems to be required for the initial contact with EF-Tu. Initial binding of the ternary complex to the ribosome is a transient step followed by further reactions in the A site. It is therefore conceivable that also the interaction between helix D of EF-Tu and the CTD of $\mathrm{L} 7 / 12$ is transient and is resolved at later steps of A-site binding, while other interactions, such as those observed by cryo-EM in the kirromycinblocked complex, ${ }^{10,11}$ are established. The functional importance of the initial binding to the flexible, four-copy ribosomal protein would be to maximize the speed of EF-Tu recruitment to the ribosome and allow an efficient turnover of the non-cognate ternary complexes, whereas the following codon-specific steps require slower mutual conformational adjustments of the ribosome, aa-tRNA, and EF-Tu. ${ }^{2,4,10,11,34}$

\section{Materials and Methods}

\section{Buffer and reagents}

Buffer A is $25 \mathrm{mM}$ Tris- $\mathrm{HCl}(\mathrm{pH} 7.5), 50 \mathrm{mM} \mathrm{NH}_{4} \mathrm{Cl}$, $10 \mathrm{mM} \mathrm{MgCl}_{2}$. Experiments were performed at $20^{\circ} \mathrm{C}$ if not stated otherwise. Chemicals were purchased from Roche Biochemicals and Merck. The mant-GTP (2'-(or$\left.3^{\prime}\right)-O-\left(N\right.$-methylanthraniloyl)guanosine $5^{\prime}$ - triphosphate) was purchased from JenaBioscienceGmbH. Ribosomes, EF-Tu, AcPhe-tRNA ${ }^{\text {Phe }}$, Phe-tRNA, and PhetRNA $^{\text {Phe }}\left(\right.$ Prf16/17) were prepared as described. ${ }^{6,35}$

\section{Mutagenesis, protein expression and purification}

Mutagenesis, protein expression and purification of EF-Tu containing a C-terminal oligohistidine tag was carried out as described. ${ }^{21}$ L7/12 was expressed as a GST-fusion protein ${ }^{36}$ and purified by affinity chromatography on glutathione-Sepharose $4 \mathrm{~B} .{ }^{27}$ Mutations were introduced by PCR mutagenesis using $P f u$ polymerase and verified by DNA sequencing. The purity of the resulting EF-Tu and L7/12 preparations was $>90 \%$ according to SDS-PAGE. To prepare ribosomes containing mutant $\mathrm{L} 7 / 12$, native $70 \mathrm{~S}$ ribosomes were depleted of $\mathrm{L} 7 / 12$ by $\mathrm{NH}_{4} \mathrm{Cl} /$ ethanol-treatment and reconstituted with an excess of purified wild-type or mutant L7/12 in buffer A containing $2 \mathrm{mM}$ DTT as described. ${ }^{18}$

\section{Preparation of ribosome and EF-Tu complexes}

Ternary complexes were formed from EF-Tu.GTP and Phe-tRNA $^{\text {Phe }}$ or Phe-tRNA ${ }^{\text {Phe }}($ Prf16/17) charged in situ; the extent of charging was $65 \%$. Ternary complexes containing Phe-tRNA ${ }^{\text {Phe }}(\operatorname{Prf16} / 17)(0.4 \mu \mathrm{M}$ prior to mixing with ribosome complexes, if not stated otherwise) were prepared by incubating $0.8 \mu \mathrm{M}$ EF-Tu, $1 \mathrm{mM}$ GTP, $3 \mathrm{mM}$ ATP, $0.6 \mu \mathrm{M}$ tRNA ${ }^{\text {Phe }}(\operatorname{Prf16} / 17), 2.5 \%$ (v/v) partially purified yeast phenylalanyl-tRNA synthetase (PheRS), $1 \mathrm{mM}$ phenylalanine, and $0.05 \mu \mathrm{M}$ EF-Ts in buffer A for 30 minutes at $37^{\circ} \mathrm{C}$. To measure rates of dipeptide formation, complexes were prepared in the same way, except that $0.4 \mu \mathrm{M}$ purified $\left[{ }^{14} \mathrm{C}\right] \mathrm{Phe}-\mathrm{tRNA}{ }^{\text {Phe }}$ was used and PheRS, ATP, and phenylalanine were omitted. When mant-GTP fluorescence was monitored, the reaction contained $0.8 \mu \mathrm{M}$ EF-Tu, $0.8 \mu \mathrm{M}$ mant-GTP, $0.4 \mu \mathrm{M}$ EF-Ts and $0.4 \mu \mathrm{M}$ purified $\left[{ }^{14} \mathrm{C}\right] \mathrm{Phe}-\mathrm{tRNA}{ }^{\mathrm{Ph}}$. To measure initial binding of ternary complex $(0.1 \mu \mathrm{M}$ before mixing) to ribosomes containing mutant L7/12, $0.2 \mu \mathrm{M}$ wild-type EF-Tu without an oligohistidine tag was incubated with $1 \mathrm{mM}$ GTP, $3 \mathrm{mM}$ ATP, $0.15 \mu \mathrm{M}$ tRNAPhe(Prf16/17), 2.5\% (v/v) PheRS, $1 \mathrm{mM}$ phenylalanine, $3 \mathrm{mM}$ phosphoenol pyruvate and $10 \mu \mathrm{g} / \mathrm{ml}$ of pyruvate kinase in buffer A containing $2 \mathrm{mM}$ DTT for 30 minutes at $37^{\circ} \mathrm{C}$.

Ribosomes were programmed with $1 \mathrm{mg} / \mathrm{ml}$ of poly(U) and a 1.2-fold excess of AcPhe-tRNA ${ }^{\text {Phe }}$ in buffer A for 15 minutes at $37^{\circ} \mathrm{C}$. All ribosome concentrations stated in the text are concentrations after mixing of the ribosome complex with ternary complex.

\section{Kinetic experiments}

Fluorescence stopped-flow measurements were performed as described ${ }^{6,25,26}$ on an SX-18MV spectrometer (Applied Photophysics). The fluorescence of proflavin was excited at $470 \mathrm{~nm}$ and measured after passing KV 500 filters (Schott). The fluorescence of 
mant-GTP was excited at $360 \mathrm{~nm}$ and measured after passing KV 408 filters (Schott). With the apparatus used, time-constants up to $500 \mathrm{~s}^{-1}$ could be measured. Experiments were performed by mixing rapidly equal volumes $(60 \mu \mathrm{l}$ each) of ternary complex and ribosome complex at the indicated concentrations. All experiments with EF-Tu mutants were performed in buffer $\mathrm{A}$. In experiments with L7/12 mutants, buffer A was supplemented with $2 \mathrm{mM}$ DTT to improve the signal-to-noise ratio at lower concentration of ternary complex containing Phe-tRNAPhe(Prf16/17). Quench-flow experiments were performed using a KinTek quench-flow apparatus under conditions identical with those employed in the stopped-flow experiments. To measure rates of dipeptide formation, equal volumes (14 $\mu \mathrm{l}$ each) of ribosome complex and ternary complex containing $\left[{ }^{14} \mathrm{C}\right]$ Phe-tRNA ${ }^{\text {Phe }}$ were mixed rapidly and, after the desired incubation time, the reaction was terminated by quenching with $0.8 \mathrm{M}$ $\mathrm{KOH}$, incubated for 30 minutes at $37^{\circ} \mathrm{C}$, neutralized, and analyzed by HPLC on a RP18 column (Merck, Darmstadt) using a gradient of acetonitrile in $0.1 \%$ $(\mathrm{v} / \mathrm{v})$ trifluoroacetic acid (TFA). ${ }^{6}$ Eluted fractions were analyzed for radioactivity by liquid-liquid scintillation counting.

\section{Determination of rate constants}

To obtain values for apparent rate constants, timecourses were evaluated by fitting to a function with a sum of two exponential terms (characterized by variable time-constants, $k_{\mathrm{app}}$, and respective amplitudes) and another variable for the final signal using TableCurve software (Jandel Scientific). Rate constants of initial binding, $k_{1}$ and $k_{-1}$, were determined from the linear concentration dependence of $k_{\text {app } 1}$. Rate constants $k_{2}$ and $k_{3}$ were calculated by fitting the concentration-dependence of the respective apparent rate constants to the following equations assuming negligibly small value for $k_{-2}:{ }^{37}$

$$
\begin{aligned}
k_{\mathrm{app} 2} & =\frac{k_{2}[\mathrm{Rs}]}{K_{\mathrm{M} 2}+[\mathrm{Rs}]}, \text { where } K_{\mathrm{M} 2}=\frac{k_{-1}+k_{2}}{k_{1}} \\
k_{\mathrm{app} 3} & =\frac{k_{\mathrm{sat}}[\mathrm{Rs}]}{K_{\mathrm{M} 3}+[\mathrm{Rs}]}, \text { where } \\
K_{\mathrm{M} 3} & =\frac{k_{-1}+k_{2}}{k_{1}} \cdot \frac{k_{3}}{k_{2}+k_{3}} \text { and } k_{\mathrm{sat}}=\frac{k_{2} \cdot k_{3}}{k_{2}+k_{3}}
\end{aligned}
$$

The combined titration data (dependencies on ribosome concentration of $k_{\mathrm{app} 2}$ and $k_{\mathrm{app} 3}$ ) for proflavin and mant observables were used, and the values of $k_{2}$ and $k_{3}$ were determined by numerical integration using Scientist for Windows software (Micromath Scientific software) with fixed values for $k_{1}$, and $k_{-1}$.

The rate constant $k_{5}$ was calculated by global fitting of the time-course of dipeptide formation using a two-step kinetic scheme as follows:

$$
\mathrm{A} \stackrel{k_{\text {delay }}}{\rightarrow} \mathrm{B} \stackrel{k_{5}}{\rightarrow} \mathrm{C}
$$

The ternary complex EF-Tu.GTP.Phe-tRNA ${ }^{\text {Phe }}$ was designated as A. The steps of initial binding, codon recognition, GTPase activation, GTP hydrolysis, and EF-Tu conformational change were grouped in the rate constant $k_{\text {delay }}$ The ribosomal complex before Phe-tRNA ${ }^{\text {Phe }}$ accommodation was designated as $\mathrm{B}$; accommodation and peptide bond formation led to the complex $C$. The rate constant $k_{4}$ was calculated from $k_{\text {delay }}$ and $k_{\text {app3 }}$ at the corresponding ribosome concentration $(1.0 \mu \mathrm{M})$ according to the transit time-approach: ${ }^{38}$

$$
\begin{gathered}
\tau_{\text {delay }}=\tau_{\text {app } 3}+\tau_{4} \\
\Rightarrow k_{4}=\frac{1}{-\frac{1}{k_{\text {app } 3}}+\frac{1}{k_{\text {delay }}}}
\end{gathered}
$$

For values that were measured directly, standard deviations were calculated from the variation of several experiments. Standard deviations for elemental rate constants were calculated using Scientist for Windows software.

\section{Acknowledgements}

We thank Wolfgang Wintermeyer for valuable comments on the manuscript, Yuri Semenkov and Vladimir Katunin for purified tRNA, and Carmen Schillings, Astrid Böhm, Simone Möbitz and Petra Striebeck for expert technical assistance. The work was supported by the Deutsche Forschungsgemeinschaft, the Alfried Krupp von Bohlen und Halbach-Stiftung, and the Fonds der Chemischen Industrie. U.K. is supported by a fellowship of the Studienstiftung des deutschen Volkes.

\section{References}

1. Rodnina, M. V., Pape, T., Savelsbergh, A., Mohr, D., Matassova, N. B. \& Wintermeyer, W. (2000). Mechanisms of partial reactions of the elongation cycle catalyzed by elongation factors Tu and G. In Ribosome: Structure, Function, Antibiotics, and Cellular Interactions (Garrett, R. A., Douthwaite, S. R., Liljas, A., Matheson, A. T., Moore, P. B. \& Noller, H. F., eds), pp. 301-317, ASM Press, Washington, DC.

2. Rodnina, M. V. \& Wintermeyer, W. (2001). Fidelity of aminoacyl-tRNA selection on the ribosome: kinetic and structural mechanisms. Annu. Rev. Biochem. 70, 415-435.

3. Rodnina, M. V., Pape, T., Fricke, R., Kuhn, L. \& Wintermeyer, W. (1996). Initial binding of the elongation factor Tu.GTP.aminoacyl-tRNA complex preceding codon recognition on the ribosome. J. Biol. Chem. 271, 646-652.

4. Ogle, J. M., Brodersen, D. E., Clemons, W. M., Jr, Tarry, M. J., Carter, A. P. \& Ramakrishnan, V. (2001). Recognition of cognate transfer RNA by the $30 \mathrm{~S}$ ribosomal subunit. Science, 292, 897-902.

5. Eccleston, J. F., Dix, D. B. \& Thompson, R. C. (1985). The rate of cleavage of GTP on the binding of PhetRNA.elongation factor Tu.GTP to poly(U)-programmed ribosomes of Escherichia coli. J. Biol. Chem. 260, 16237-16241.

6. Pape, T., Wintermeyer, W. \& Rodnina, M. V. (1998). Complete kinetic mechanism of elongation factor Tu-dependent binding of aminoacyl-tRNA to the A site of the E. coli ribosome. EMBO J. 17, 7490-7497.

7. Rodnina, M. V., Fricke, R., Kuhn, L. \& Wintermeyer, W. (1995). Codon-dependent conformational change of elongation factor Tu preceding GTP hydrolysis on the ribosome. EMBO J. 14, 2613-2619.

8. Stark, H., Rodnina, M. V., Rinke-Appel, J., Brimacombe, R., Wintermeyer, W. \& van Heel, M. 
(1997). Visualization of elongation factor Tu on the Escherichia coli ribosome. Nature, 389, 403-406.

9. Valle, M., Sengupta, J., Swami, N. K., Grassucci, R. A., Burkhardt, N., Nierhaus, K. H. et al. (2002). Cryo-EM reveals an active role for aminoacyl-tRNA in the accommodation process. EMBO J. 21, 3557-3567.

10. Stark, H., Rodnina, M. V., Wieden, H.-J., Zemlin, F., Wintermeyer, W. \& van Heel, M. (2002). Ribosome interactions of aminoacyl-tRNA and elongation factor $\mathrm{Tu}$ in the codon recognition complex. Nature Struct. Biol. 9, 849-854.

11. Valle, M., Zavialov, A., Li, W., Stagg, S. M., Sengupta, J., Nielsen, R. C. et al. (2003). Incorporation of aminoacyl-tRNA into the ribosome as seen by cryoelectron microscopy. Nature Struct. Biol. 10, 899-906.

12. Traut, R. R., Dey, D., Bochkariov, D. E., Oleinikov, A. V., Jokhadze, G. G., Hamman, B. \& Jameson, D. (1995). Location and domain structure of Escherichia coli ribosomal protein L7/L12: site specific cysteine cross- linking and attachment of fluorescent probes. Biochem. Cell Biol. 73, 949-958.

13. Yusupov, M. M., Yusupova, G. Z., Baucom, A., Lieberman, K., Earnest, T. N., Cate, J. H. \& Noller, H. F. (2001). Crystal structure of the ribosome at $5.5 \AA$ resolution. Science, 292, 883-896.

14. Ban, N., Nissen, P., Hansen, J., Capel, M., Moore, P. B. \& Steitz, T. A. (1999). Placement of protein and RNA structures into a 5 A-resolution map of the $50 \mathrm{~S}$ ribosomal subunit. Nature, 400, 841-847.

15. Ban, N., Nissen, P., Hansen, J., Moore, P. B. \& Steitz, T. A. (2000). The complete atomic structure of the large ribosomal subunit at $2.4 \AA$ resolution. Science, 289, 905-920.

16. Bernabeu, C., Vazquez, D. \& Ballesta, J. P. (1976). Activities of protein-deficient particles derived from 50-S ribosomal subunits by $\mathrm{NH}_{4} \mathrm{Cl} /$ ethanol treatment. Eur. J. Biochem. 69, 233-241.

17. Möller, W., Schrier, P. I., Maassen, J. A., Zantema, A., Schop, E., Reinalda, H. et al. (1983). Ribosomal proteins L7/L12 of Escherichia coli. Localization and possible molecular mechanism in translation. J. Mol. Biol. 163, 553-573.

18. Mohr, D., Wintermeyer, W. \& Rodnina, M. V. (2002). GTPase activation of elongation factors Tu and $\mathrm{G}$ on the ribosome. Biochemistry, 41, 12520-12528.

19. Wang, Y., Jiang, Y. X., Meyering-Voss, M., Sprinzl, M. \& Sigler, P. B. (1997). Crystal structure of the EF-Tu.EF-Ts complex from Thermus thermophilus. Nature Struct. Biol. 4, 650-656.

20. Kawashima, T., Berthet-Colominas, C., Wulff, M., Cusack, S. \& Leberman, R. (1996). The structure of the Escherichia coli EF-Tu.EF-Ts complex at $2.5 \AA$ resolution. Nature, 379, 511-518.

21. Wieden, H. J., Gromadski, K., Rodnin, D. \& Rodnina, M. V. (2002). Mechanism of elongation factor (EF)-Tscatalyzed nucleotide exchange in EF-Tu. Contribution of contacts at the guanine base. J. Biol. Chem. 277, 6032-6036.

22. Wieden, H. J., Wintermeyer, W. \& Rodnina, M. V. (2001). A common structural motif in elongation factor Ts and ribosomal protein L7/12 may be involved in the interaction with elongation factor Tu. J. Mol. Evol. 52, 129-136.

23. Rodnina, M. V., Fricke, R. \& Wintermeyer, W. (1994). Transient conformational states of aminoacyl-tRNA during ribosome binding catalyzed by elongation factor Tu. Biochemistry, 33, 12267-12275.

24. Piepenburg, O., Pape, T., Pleiss, J. A., Wintermeyer, W., Uhlenbeck, O. \& Rodnina, M. V. (2000). Intact aminoacyl-tRNA is required to trigger GTP hydrolysis by elongation factor $\mathrm{Tu}$ on the ribosome. Biochemistry, 39, 1734-1738.

25. Knudsen, C., Wieden, H. J. \& Rodnina, M. V. (2001). The importance of structural transitions of the switch II region for the functions of elongation factor $\mathrm{Tu}$ on the ribosome. J. Biol. Chem. 276, 22183-22190.

26. Pape, T., Wintermeyer, W. \& Rodnina, M. V. (1999). Induced fit in initial selection and proofreading of aminoacyl-tRNA on the ribosome. $E M B O \mathrm{~J} . \mathbf{1 8}$, 3800-3807.

27. Savelsbergh, A., Katunin, V. I., Mohr, D., Peske, F., Rodnina, M. V. \& Wintermeyer, W. (2003). An elongation factor G-induced ribosome rearrangement precedes tRNA-mRNA translocation. Mol. Cell, 11, 1517-1523.

28. Kirsebom, L. A. \& Isaksson, L. A. (1985). Involvement of ribosomal protein L7/L12 in control of translational accuracy. Proc. Natl Acad. Sci. USA, 82, 717-721.

29. Kirsebom, L. A., Amons, R. \& Isaksson, L. A. (1986). Primary structures of mutationally altered ribosomal protein L7/L12 and their effects on cellular growth and translational accuracy. Eur. J. Biochem. 156, 669-675.

30. Leijonmarck, M. \& Liljas, A. (1987). Structure of the C-terminal domain of the ribosomal protein L7/L12 from Escherichia coli at $1.7 \AA$ A. J. Mol. Biol. 195, 555-579.

31. Wahl, M. C., Bourenkov, G. P., Bartunik, H. D. \& Huber, R. (2000). Flexibility, conformational diversity, and two dimerization modes in complexes of ribosomal protein L12. EMBO J. 19, 174-186.

32. Hernandez, F., de No, C. \& Palacian, E. (1984). Functional implication of the sole arginine residue of ribosomal proteins L7/L12. Mol. Biol. Rep. 10, 75-78.

33. Koteliansky, V. E., Domogatsky, S. P., Gudkov, A. T. \& Spirin, A. S. (1977). Elongation factor-dependent reactions of ribosomes deprived of proteins L7 and L12. FEBS Letters, 73, 6-11.

34. Ogle, J. M., Murphy, F. V., Tarry, M. J. \& Ramakrishnan, V. (2002). Selection of tRNA by the ribosome requires a transition from an open to a closed form. Cell, 111, 721-732.

35. Rodnina, M. V. \& Wintermeyer, W. (1995). GTP consumption of elongation factor Tu during translation of heteropolymeric mRNAs. Proc. Natl Acad. Sci. USA, 92, 1945-1949.

36. Savelsbergh, A., Mohr, D., Wilden, B., Wintermeyer, W. \& Rodnina, M. V. (2000). Stimulation of the GTPase activity of translation elongation factor $\mathrm{G}$ by ribosomal protein L7/12. J. Biol. Chem. 275, 890-894.

37. Cleland, W. W. (1975). Partition analysis and the concept of net rate constants as tools in enzyme kinetics. Biochemistry, 14, 3220-3224.

38. Fersht, A. (1998). Structure and Mechanism in Protein Science, WH Freeman, New York.

Edited by J. Doudna 